(C) [2008] IEEE. Reprinted, with permission, from [Moha'med O. Al-Jaafreh and Adel A. Al-Jumaily, Type-2 Fuzzy System Based Blood Pressure Parameters Estimation, Modeling \& Simulation, 2008. AICMS 08. Second Asia International Conference on 13-15 May 2008]. This material is posted here with permission of the IEEE. Such ermission of the IEEE does not in any way imply IEEE endorsement of any of the University of Technology, Sydney's products or services. Internal or personal use of this material is permitted. However, permission to reprint/republish this material for advertising or promotional purposes or for creating new collective works for resale or redistribution must be obtained from the IEEE by writing to pubs-permissions@ieee.org. By choosing to view this document, you agree to all provisions of the copyright laws protecting it 


\section{Type-2 Fuzzy System Based Blood Pressure Parameters Estimation}

\author{
Moha'med O. Al-Jaafreh \\ Faculty of Engineering, University of \\ Technology, Sydney, Australia \\ maljaaf@eng.uts.edu.au
}

\author{
Adel A. Al-Jumaily \\ Faculty of Engineering, University of \\ Technology, Sydney, Australia \\ adel@eng.uts.edu.au
}

\begin{abstract}
Blood pressure parameters; Systolic, Diastolic and Mean Blood Pressure, have high correlation relationship with heart rate, but this relationship is nonlinear, multimode, and ambiguity. Type-2 Fuzzy is an intelligent technique and it has high capability to deal with the problem with high uncertainty that blood pressure parameters with heart rate relationship can be classify under them.

This paper presents an Interval Type-2 Fuzzy System to estimate Systolic, Diastolic and Mean blood pressure; based on the relationship between systolic, diastolic and mean blood pressures and heart rate.

The Interval Type-2 Fuzzy System is used to estimate systolic, diastolic and mean blood pressures values of thirty cases. The results are compared with real reading of systolic, diastolic and mean blood pressure and very encouraging results are achieved; the absolute value of mean of difference between real systolic, diastolic and mean blood pressures and estimated systolic, diastolic and mean blood pressures are less than $5 \mathrm{mmHg}$.
\end{abstract}

\section{Introduction}

Blood pressure parameters (BPPs); Systolic Blood Pressure (SBP), Diastolic Blood Pressure (DBP) and Mean Arterial blood Pressure (MAP), are very important to diagnose subjects' health situations and help Physicians to prevent subjects from many cardiovascular diseases; such as, hypertension, asthma and heart attack.

BPPs are measured by Cannulation and Auscultatory methods; Cannulation method measures blood pressure directly, continuously and accurately, but it is invasive, while Auscultatory method measures BPPs by applying external and uncomfortable pressure by cuff, cuff inflation and deflation is time consuming and non-continuous [1]. Hence, current researchers try to establish and invent a method to measure blood pressure parameters by continuous, comfortable, noninvasive, reliable, and user friendly sensor by using photo-plethysmography (PPG) sensor.

Recently, in 2005, Yan and Zhang used PPG sensor with electrocardiograph (ECG) to estimate BPPs and they stated that the normalized harmonic area of a discrete period transform of PPG signal has high correlation with BP [2].

Moreover, Ma and Zhang stated that the time interval between peak of electro-cardiograph (ECG) and the front foot of PPG signal, known as the $\mathrm{PTT}_{\mathrm{f}}$; has high correlation with BP variability [3]. Furthermore, Poon and Zhang has found a model to estimate BP by using a weighed PTT $\left(\mathrm{PTT}_{\mathrm{w}}\right)$; this feature is dependent on individual subject health situation. $\mathrm{PTT}_{\mathrm{w}}$ depends on calibrating measurements of SBP and DBP [4].

These methods depend on two sensors to estimate BPPs; which increase the probability of errors' occurring and need improvements and modifications to estimate reliable BPPs.

In this paper, BPPs are estimated depend on PPG sensor only and Interval Type-2 Fuzzy system (IT2FS). PPG sensor is used to estimate Heart rate (HR), then the HR value is processed by IT2FS to produce the values of BPPs; SBP, DBP and MAP.

This paper is organized as follows; section two introduces and highlights Interval Type-2 Fuzzy system, section three presents the methodology of designing IT2FS to estimate BPPs, database and results of IT2FS are illustrated in tables and figures in section four, finally the paper is concluded in section five. 


\section{Interval Type-2 Fuzzy system}

Fuzzy set theory was inspired by Professor L. A. Zadeh [5], in 1965, to effectively and quantitatively deal with nature problems. Fuzzy sets theory has set the principle for computing by words; Collecting people words about any problem produce different levels of uncertainty according to their perspectives, outlooks and experiences.

Fuzzy sets include some of uncertainty levels by generalizing 0 and 1 membership values of a crisp set to a membership function (MF) of a fuzzy set; as shown in Fig.1, the relationship between age and "young" is represented by a crisp value that either 0 or 1; 0 means no association at all and 1 indicates complete association, while, in Fuzzy set theory, the "young" association membership set is generalized to MF where age 0-10 is "young" with membership value 1 , age 30 with membership value 0.75 and age 50 with membership value 0.1 as shown in Fig.2 [6].

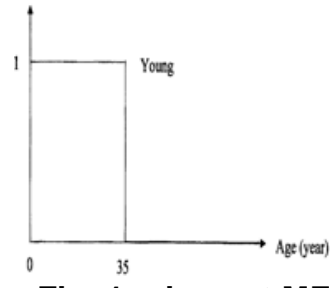

Fig. 1 crisp set MF

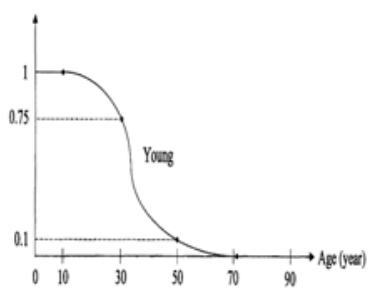

Fig. 2 Type-1 MF
In 1975, the motivation of type-2 fuzzy set was extended by Zadeh [7] of type-1 fuzzy set. Then, Mizumoto and Tanaka presented some properties of fuzzy sets of type-2 [8]. Later In 1998, Karnik and Mendel introduced T2 FS comprehensively [9].

T2 FS model consists of four parts; firstly, Fuzzifier that maps the input and the output into T2 fuzzy sets by PMFs and initializes input and output PMFs' parameters. Secondly, “IF ..., THEN ..." rules to connect fuzzified sets of input with fuzzified sets of output. Thirdly, inference engine which combines the rules and forms a mapping between the input sets and the output sets. Finally, a Deffuzification process to produce crisp values of output by two stages; type reduction to descend type-2 set to type-1 set then defuzzifys type- 1 set to crisp value. The block diagram of T2 FS is illustrated in Fig. 3 [10].

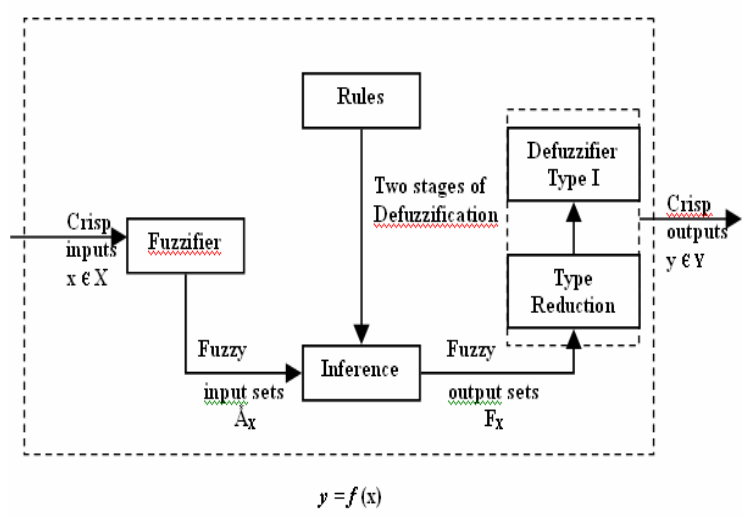

Fig. 3. Block diagram of Type-2 Fuzzy System

T2 FS is expanded of type- 1 FS to include the effects of more different levels of uncertainties in rulebase of FS. T2 FS is characterized by a $\mu_{\tilde{A}}(x)$ as Primary Membership Function (PMF) for set $\tilde{\tilde{A}}$ and the membership value for each element of $\tilde{\mathrm{A}}$ is fuzzy set; which is called secondary membership function or membership grade.

Gaussian distribution is most common distribution used in FS to include the uncertainty levels and express the collection of people words about any problem according to their perspectives and experiences.

By moving Gaussian type-1 MF to left and right in a non-uniform manner Gaussian type-2 PMF is formed as shown in Fig. 4.
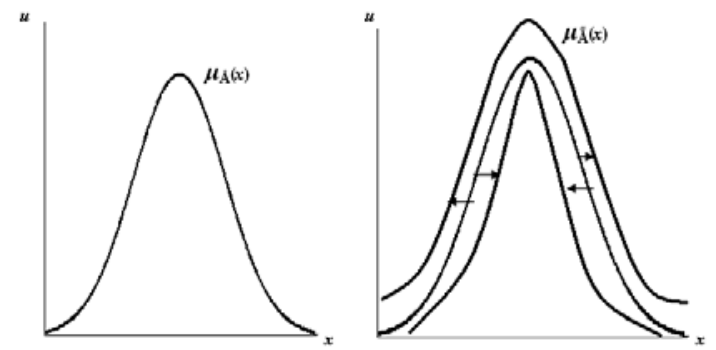

Gaussian Type-1 MF Gaussian Type-2 PMF Fig. 4. Extension Type-1 MF to Type-2 PMF

Gaussian Interval T2 FS is proposed as practical case of general Gaussian T2 FS to avoid terrible and horrendous, as well as, sides of $\mathrm{T} 2 \mathrm{MF}$ are bounded by Upper MF and Lower MF to form Interval T2 PMF and the Footprint of Uncertainty (FOU) as shown in Fig. 5. 


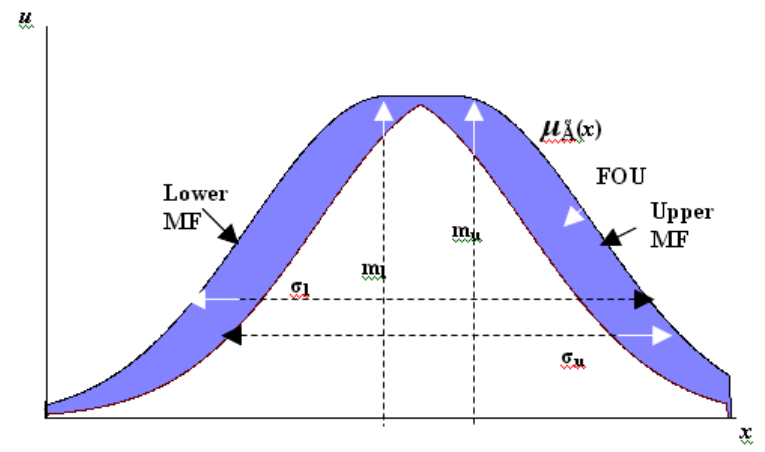

Fig. 5. Gaussian Interval Type-2 PMF

As illustrated in Fig. 5, Gaussian Interval T2 PMF parameters are:

1- Lower mean $\mathrm{m}_{1}$.

2- Upper mean $\mathrm{m}_{\mathrm{u}}$.

3- Lower standard deviation $\sigma_{\mathrm{l}}$.

4- Upper standard deviation $\sigma_{\mathrm{u}}$.

These parameters control the shape of primary membership functions of IT2 FS.

IT2FS is trained by database of input variables and output variables to minimize the error between the estimated output of FS and the real output by adjusting the input and output PMFs' parameters.

Afterwards the designed FS is used to estimate and predict output variables depend on input variables.

\section{Methodology:}

In this paper, Blood Pressure Parameters (BPPs); systolic, diastolic and mean pressures are estimated depend on photo-plethysmography (PPG) sensor and intelligent Interval Type-2 Fuzzy system (IT2FS).

PPG sensor is attached with subject's pointer finger, as shown in Fig. (6), to compute subject's Heart Rate (HR) from the frequency of PPG signal by using this equation (1) [11]:

$$
\mathrm{HR}=60 \times f
$$

Where $f$ is frequency of PPG signal.

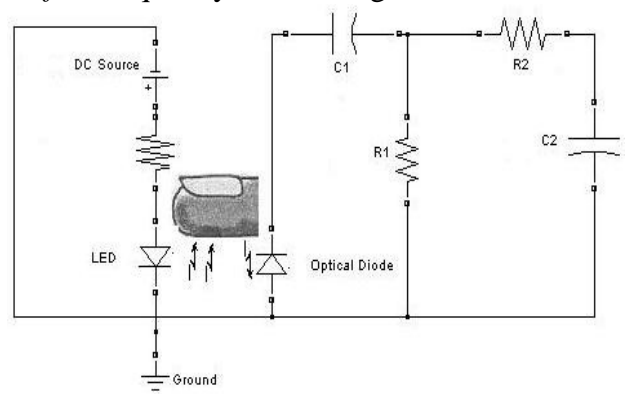

Fig. 6 Circuit Model of PPG sensor

On other hand, Systolic Blood Pressure (SBP) and Diastolic Blood Pressure (DBP) are estimated by different IT2FS; where the computed HR of equation
(1) is the input for both IT2FSs, then the output of two IT2FSs; SBP and DBP are combined to calculate Mean Arterial blood Pressure (MAP) by equation (2) [1]:

$$
\mathrm{MAP}=\mathrm{DBP}+\frac{1}{3} \times(\mathrm{SBP}-\mathrm{DBP})---(2)
$$

IT2FS is designed by following these steps:

1. The computed HR of equation (1); input of both IT2FSs, is fuzzified to five singleton Gaussian Interval Type-2 (Type-1) PMFs that take linguistic expressions; very Low, Low, Normal, High and very High as shown in Fig. 7. And the PMFs' parameters for $\mathrm{HR}$ are initialized according to American Health Association (AHA) standards [12].

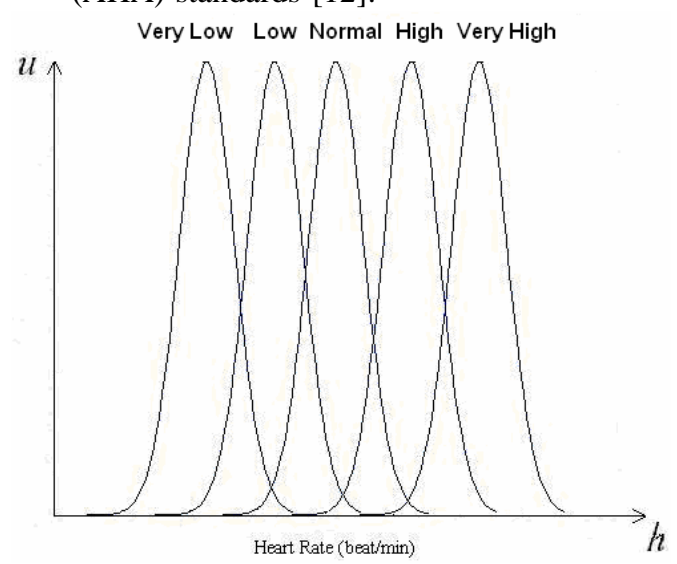

Fig. 7. Fuzzification of Heart Rate to five singleton Interval Type-2 fuzzy sets

2. Systolic and Diastolic blood pressures are represented by five Gaussian Interval type-2 PMFs that take linguistic expressions; very Low, Low, Normal, High and very High as shown in Fig. 8. And the PMF parameters for SBP and DBP are initialized according to AHA standards [12].

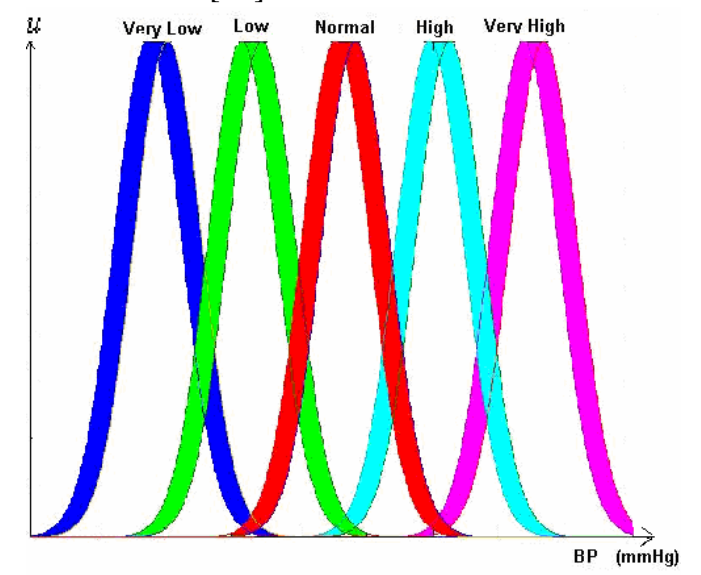

Fig. 8: Type-2 fuzzy sets to represent output values of each SBP and DBP 
3. Fuzzy rules are located as one rule for each fuzzy set, where each of the five rules is:

IF HR is $\tilde{A}$, THEN BBP is $\breve{G}$

4. Inference engine combines the rules, forms a mapping between HR fuzzy sets and BBPs' fuzzy sets and finds where HR has non-zero membership function values.

5. Defuzzification process is implemented by following two steps:

a- Descend the Type-2 membership values by computing upper MF $\mathrm{y}_{\mathrm{r}}$ by equation (3):

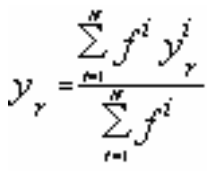

$y_{r}$ upper MF of output

$i$ is variable value changed from 1 to $N$

$N$ is number of non-zero membership function values created by firing the rules

$f^{i}$ is fuzzy value of input of $i$

$y_{r}^{i}$ is mean value of output upper MF

and computing lower MF $\mathrm{y}_{\mathrm{l}}$ by equation (4):

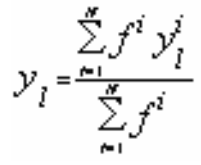

$y_{l}$ is lower MF of output

$y_{l}^{i}$ is mean value of output lower MF

b- compute the output of IT2FS by using Centre of Sums as defuzzifiction method as in equation (5):

$$
y=\frac{y_{i}+y_{r}}{2}
$$

$\mathrm{y}$ is output of Fuzzy system [13].

Designed IT2FSs are trained by database of 30 subjects, database criteria and results of IT2FSs are illustrated in next section.

\section{Results}

Three readings of blood pressure parameters heart rates of Adult healthy 30 subjects were taken by digital automatic blood pressure monitor, "OMRON IAIB Model HEM-7000-C1L”, made by OMRON Healthcare Japan [14].

The thirty subjects are including twenty four females and six males, aged between 17-68 years old, weighted (W.) between 45-162 kg, are students at
Adult Migrant English Programme, organised by Macquarie Community College, Australia.

The average of heart rate readings and systolic and diastolic blood pressures readings of each subject are given in table 1.

Table 1: Average of $H R$ and $B P$ readings of 30 subjects

\begin{tabular}{|r|l|l|l|r|r|r|}
\hline No & Age & Sex & W. & \multicolumn{1}{l|}{ SBP } & DBP & HR \\
\hline & Years & M/F & kg & mmHg & mmHg & b/m \\
\hline 1 & 68.00 & F & 92.00 & 121.33 & 84.67 & 76.00 \\
\hline 2 & 42.00 & F & 64.00 & 125.33 & 80.33 & 70.67 \\
\hline 3 & 24.00 & F & 60.00 & 79.33 & 50.00 & 86.00 \\
\hline 4 & 38.00 & F & 77.00 & 122.67 & 74.00 & 84.00 \\
\hline 5 & 27.00 & F & 55.00 & 105.33 & 73.67 & 90.67 \\
\hline 6 & 24.00 & F & 45.00 & 97.33 & 64.33 & 68.67 \\
\hline 7 & 25.00 & F & 50.00 & 110.00 & 68.67 & 74.33 \\
\hline 8 & 24.00 & F & 52.00 & 119.67 & 77.33 & 82.33 \\
\hline 9 & 22.00 & F & 45.00 & 125.67 & 78.33 & 79.67 \\
\hline 10 & 34.00 & F & 65.00 & 108.00 & 71.33 & 66.33 \\
\hline 11 & 61.00 & F & 74.00 & 112.00 & 72.00 & 63.33 \\
\hline 12 & 59.00 & F & 75.00 & 156.33 & 89.33 & 88.00 \\
\hline 13 & 57.00 & F & 64.00 & 141.67 & 77.33 & 74.00 \\
\hline 14 & 28.00 & M & 68.00 & 114.00 & 71.33 & 86.00 \\
\hline 15 & 48.00 & M & 80.00 & 124.00 & 85.67 & 83.67 \\
\hline 16 & 25.00 & F & 72.00 & 122.67 & 85.67 & 77.33 \\
\hline 17 & 27.00 & F & 53.00 & 122.67 & 92.00 & 88.67 \\
\hline 18 & 35.00 & F & 50.00 & 110.00 & 82.00 & 70.33 \\
\hline 19 & 23.00 & F & 55.00 & 120.33 & 78.67 & 88.00 \\
\hline 20 & 22.00 & F & 63.00 & 99.33 & 60.67 & 85.67 \\
\hline 21 & 20.00 & F & 162.0 & 96.33 & 63.00 & 79.33 \\
\hline 22 & 25.00 & F & 66.00 & 100.67 & 58.67 & 87.67 \\
\hline 23 & 26.00 & M & 92.00 & 112.67 & 75.33 & 76.00 \\
\hline 24 & 51.00 & F & 70.00 & 110.00 & 78.67 & 71.00 \\
\hline 25 & 26.00 & F & 58.00 & 107.33 & 78.00 & 83.67 \\
\hline 26 & 32.00 & F & 73.00 & 104.00 & 76.67 & 100.3 \\
\hline 27 & 49.00 & F & 63.00 & 144.00 & 83.33 & 88.67 \\
\hline 28 & 19.00 & F & 55.00 & 105.00 & 72.33 & 72.00 \\
\hline 29 & 17.00 & M & 72.00 & 118.67 & 70.33 & 67.00 \\
\hline 30 & 23.00 & M & 65.00 & 129.00 & 85.00 & 79.00 \\
\hline
\end{tabular}

Twenty subjects' readings are used to train designed IT2FS, the PMFs' parameters, as shown in Fig. 5, are adjusted within AHA standards, to achieve smallest errors between real and estimated SBP and DBP.

The trained IT2FSs are used to estimate SBP and DBP for remaining ten subjects and MAP are computed by equation (2). 
To show the good performance of IT2FSs; our estimated values of BPPs are compared with real values of BPPs and the Mean and Standard Deviation (SD) of the difference between the real and estimated SBP, DBP and MAP for remain ten subjects are given in table 2:

Table 2: Mean and SD of the difference between the real and estimated values

\begin{tabular}{|c|c|c|c|}
\hline & $\begin{array}{c}\text { SBP } \\
(\mathrm{mmHg})\end{array}$ & $\begin{array}{c}\text { DBP } \\
(\mathrm{mmHg})\end{array}$ & $\begin{array}{c}\text { MAP } \\
(\mathrm{mmHg})\end{array}$ \\
\hline mean & 3.8 & -5.0 & 4.6 \\
\hline SD & 13.8 & 8.6 & 10.3 \\
\hline
\end{tabular}

In addition, the Bland and Altman [15] plot method is used to show graphically; how estimated values of BPPs are close to real values of BPPs, the Bland and Altman plot of SBP, DBP and MAP are shown in Fig. 9, Fig. 10 and Fig. 11, respectively.

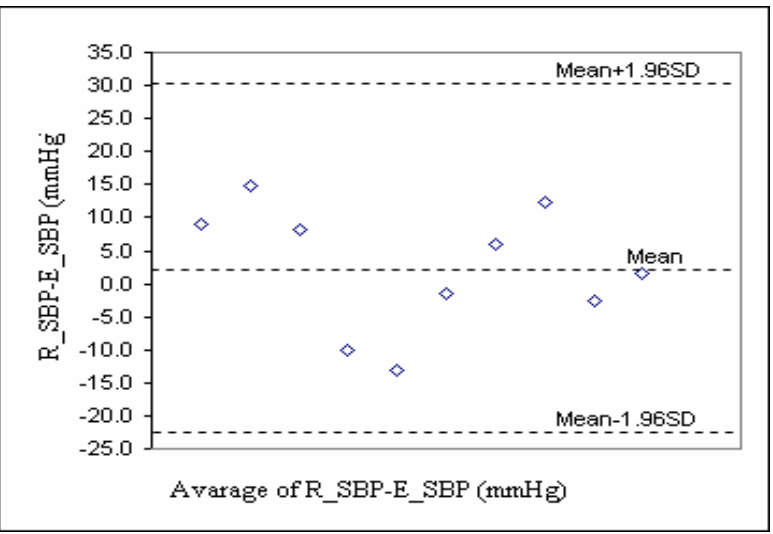

Fig. 9. Bland Altman plot of SBP

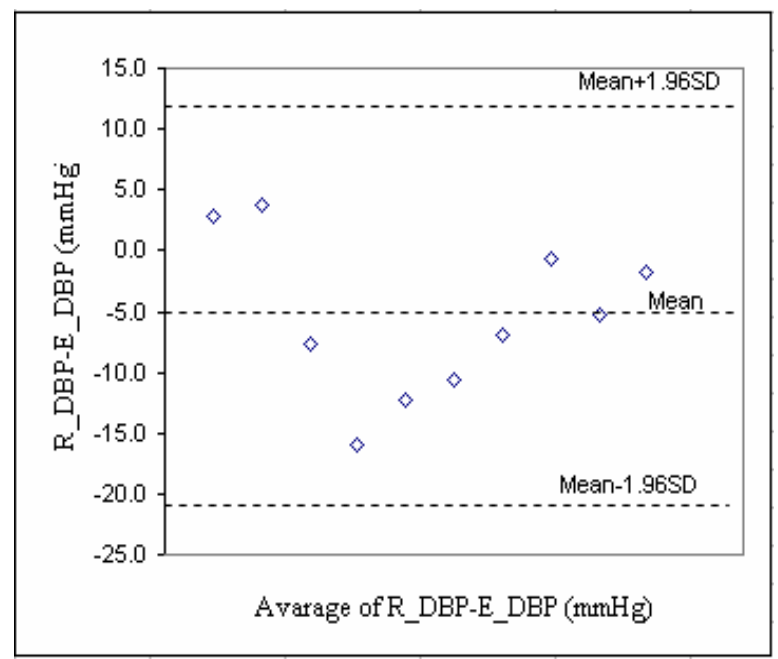

Fig. 10. Bland Altman plot of DBP

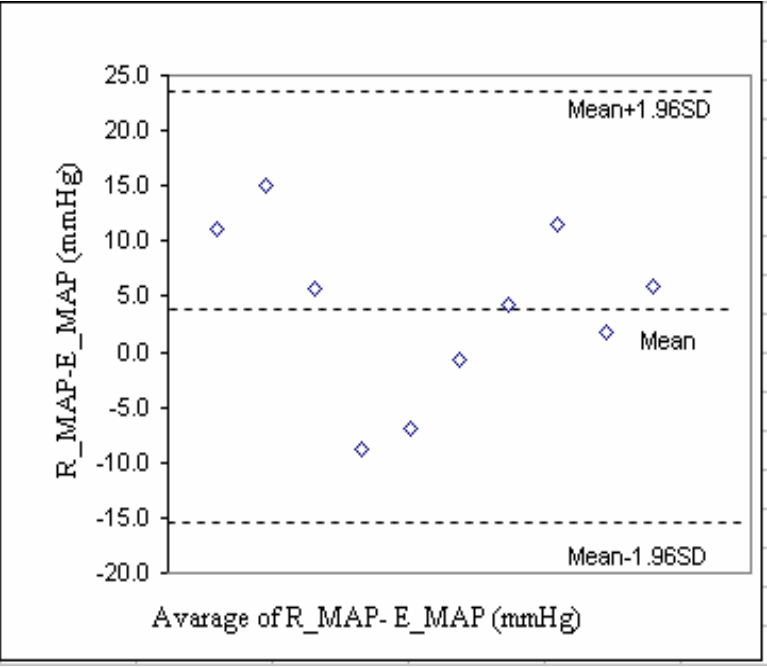

Fig. 11. Bland Altman plot of MAP

In Bland Altman plots of SBP, DBP and MAP, all estimated values are located close to mean of difference and within the border of (mean $\pm 1.96 \mathrm{SD}$ ), hence, IT2FSs achieve good and near accurate results.

\section{Conclusions}

This paper proposed an Interval Type-2 Fuzzy System to estimate Blood pressure parameters; SBP, DBP and MAP; based on Heart rate, which is measured by as photo-plethysmography (PPG) sensor.

The estimated values of SBP, DBP and MAP are compared with real SBP, DBP and MAP and very encouraging results are achieved; the absolute value of mean of difference between Real SBP, DBP and MAP and Estimated SBP, DBP and MAP are less than 5 $\mathrm{mmHg}$.

Moreover, all estimated values of SBP, DBP and MAP are located close to mean of difference and within the border of Bland \& Altman plot; (mean \pm $1.96 \mathrm{SD})$.

In Future work, designed IT2FSs for BPPs can be programmed into a microcontroller and a PPG sensor can be integrated to acquire direct $\mathrm{HR}$ from the subjects and to facilitate the measurements of blood pressure parameters.

\section{Acknowledgement}

The authors would like to thank Mr. Osman Mahmood and his team for recording the readings of the thirty subjects. 


\section{References}

[1] O'Brien E. and O'Malley K., Essentials of blood pressure measurement, with a foreword by George E. Burch Edinburgh; New York: Churchill Livingstone, 1981.

[2] Y. Yan and Y. Zhang, Noninvasive Estimation of Blood Pressure Using Photoplethysmographic Signals in the Period Domain, Engineering in Medicine and Biology Society, Conference Proceedings, 27th Annual International Conference, Shanghai, China, Sept. 1-4, 2005. pp 3583 - 3584.

[3] T. Ma and Y. Zhang, A Correlation Study on the Variabilities in Pulse Transit Time, Blood Pressure, and Heart Rate Recorded Simultaneously from Healthy Subjects, Engineering in Medicine and Biology Society, Conference Proceedings, 27th Annual International Conference, Shanghai, China, Sept. 1-4, 2005. pp. 996 - 999.

[4] C. Poon and Y. Zhang, Cuff-less and Noninvasive Measurements of Arterial Blood Pressure by Pulse Transit Tim, Engineering in Medicine and Biology Society, Conference Proceedings, 27th Annual International Conference, Shanghai, China, Sept. 1-4, 2005. pp. 5877 - 5880.

[5] L.A. Zadeh, "Fuzzy sets", Information and Control, vol.8, pp.: 338- 352, 1965.

[6] H. Ying, "Fuzzy Control and Modeling: Analytical Foundations and Applications”, WileyIEEE Press, pp.:1-13, Basic Fuzzy Mathematics for Fuzzy Control and Modeling. Ch.1, 2000.

[7] L. A. Zadeh, "The concept of a linguistic variable and its application to approximate reasoning," Information Sciences, vol. 8, pp.: 199-249, 1975.
[8] M. Mizumoto and K. Tanaka, "Some properties of fuzzy sets of type 2," Information and Control, vol. 31, pp.: 312-340, 1976.

[9] N. Karnik and J. M. Mendel, "Introduction to type-2 fuzzy logic systems”, Proceedings of IEEE International Conference on World Congress on Computational Intelligence, vol.2, pp.: 915-920, 1998.

[10] J. M. Mendel, R. I. John, and F. Liu, "Interval Type-2 Fuzzy Logic Systems Made Simple," IEEE Transactions on Fuzzy Systems, vol. 14, pp.: 808-821, 2006.

[11] G.Y. Jeong, K.H. Yu, and N.G. Kim, “Continuous Blood Pressure Monitoring using Pulse Wave Transit Time”, ICCAS2005 Conference, KINTEX, Gyeonggi-Do, Korea , June 2-5, 2005.

[12] American heart association (AHA), 2007, Available on line at: http://www.americanheart.org/.

[13] J. M. Mendel, "Uncertain rule-based fuzzy logic systems: introduction and new directions”, Prentice Hall PTR, Upper Saddle River, New Jersey, USA, 2001.

[14] Omron Healthcare, Inc., 2007, Blood Pressure Monitor, Omron healthcare, Inc. Japan, [online], accessed on $15^{\text {th }}$ June 2007, available at http://www.omronhealthcare.com.

[15] J. M. Bland, D. G. Altman (1999) Measuring agreement in method comparison studies. Statistical Methods in Medical Research, 8, 135160. 